\title{
Reviews
}

\section{MRS Census and Geodemographics Group annual conference: Harnessing open data for business advantage}

\author{
Journal of Direct, Data and Digital Marketing Practice (2015) 16, \\ 236-238. doi:10.1057/dddmp.2015.6
}

\section{5 years of promoting the Census}

\section{Visualising the richness of Census data}

\section{Shopping demands - \\ work, rest and play}

The clue is in the name
The main aim of this seminar was to show how users have been applying Census and other open data sources to a variety of business and research applications. The seminar was expertly chaired by Peter Mouncey, editor-inchief of the International Journal of Market Research. Mouncey pointed out that the Census and Geodemographics Group (CGG) was celebrating its 25th anniversary this year. For his introduction to the day, he reminded delegates of how the CGG was formed, back in the late 1980s, and identified the role that CGG has played in promoting the use of Census and geodemographic information and working with the Office of National Statistics (ONS) and others to represent the needs of the research industry.

The opening presentation was given by Alan Smith from ONS, who delivered a series of examples of advanced ways in which data can be presented and visualized. Smith commented that a recent IPSOS MORI survey had measured 'emotional innumeracy' and found that people are generally poor statisticians. Therefore, data providers such as ONS need to do a better job of dispelling common misconceptions. He demonstrated a website that presents a simple quiz on 'How well do you know your area?' This site had received a quarter of a million hits in the first 4 days from launch, illustrating the usefulness of gamification as a tool for making statistics interesting.

Graham Smith of CACI focused on the need to employ a wide range of data sources when carrying out analysis in the retail sector. He illustrated his talk using case studies on site location for the Southern Co-Operative and on identifying the hottest postcodes for convenience stores, done for The Grocer. Smith identified the most useful open sources for analysing retail demand among workers in an area, including Census workday population and workplace statistics as well as the Business Register Employment Survey (BRES). He closed by recommending that a 'fitness for purpose' approach should be taken, that is, open data should not be used simply because it happens to be freely available, and that a combination of sources can sometimes give the best results.

\section{Convenience store catchment area modelling}

Tim Drye of DataTalk highlighted the complexity of modelling convenience store catchment areas and demonstrated a two-stage approach that enables brands and retailers to target products and promotions more 


\section{Why look at who moves where?}

\section{Geodemographics of internet use and engagement}

\section{Open data update}

\section{Report from the open data coalface}

accurately in convenience stores. The first stage was to understand the local contexts of stores, and his analysis had identified seven types of store locations and formed a profile of each type. The second stage was to understand the people who frequent each type of location; therefore, Drye had created a segmentation of consumer types and analysed their relationships with the location clusters. By overlaying sales data, the most successful combinations could then be identified.

\section{Census migration and commuting data}

Mike Coombes from Newcastle University first explained the features of flow data from the Census, enabling analysis of commuting flows from home to workplace, and migration flows from previous home to current home. He showed how the commuting flow data had been used to create travel-to-work areas (TTWA) that define local labour markets, and illustrated the results with a series of TTWA maps. Coombes then discussed the creation of housing market areas that partition England into zones based on migration patterns and illustrated how distance of move varies between owner occupiers and renters. He closed by commenting on potential future applications, including work by University College London with Sainsbury's on mapping patterns of cultural identity and heritage using open data.

Alex Singleton from Liverpool University demonstrated the marked differences in internet usage by geodemographic types and illustrated the e-society classification that mapped these differences back in 2006. However, subsequent changes in infrastructure (eg, growth in broadband, $4 \mathrm{G}$ network) and user behaviour (eg, use of social networks, internet access via smartphones) had necessitated the development of a new internet user classification. This had been built using a similar methodology to the 2011 output area classification, and further work was currently in progress to refine its use for retail catchment area analysis.

Nick Halliday from the National Audit Office delivered an update on open data developments on behalf of the Open Data User Group (ODUG). Halliday explained that one of ODUG's main priorities over the last year had been to support the development of the government's national information infrastructure (NII). The intention was that the NII would contain the data held by the government that is likely to have the broadest and most significant economic impact if made available and accessible outside of government. ODUG had been reviewing what the value of the NII should be and which data should be included in it - the initial approach to identifying NII datasets had been quite limited, in ODUG's view.

Blair Freebairn of Geolytix put forward the view that open data was capable of transforming the way that the world does business, citing as an example the data that would be needed for 3D printing to become 'mainstream'. He explained that the value of open data would come from combining multiple sources and mentioned a number of examples, including building a geography of place names, identifying retail 'hot spots', creating a routable road network and developing small area demand estimates using raw Census data. Freebairn felt that the decision to proceed 


\author{
Harnessing Census \\ microdata
}

\section{Social and cultural influences in the digital age}

\section{Continued value of \\ Census data}

with the 2021 Census was great news, however, there will also be new open data sources that will help to change and enhance it.

Barry Leventhal from BarryAnalytics introduced the concept of microdata - samples of raw data on households and individuals drawn from the Census - and summarized its history in the United Kingdom. Following the 2011 Census, microdata products have been produced at three levels of access: public use, safeguarded and secure. For business users, the safeguarded product is likely to be of greatest interest; however, access may be more difficult. Leventhal presented case study examples of applying microdata and summarized the main use cases in market research.

Richard Webber of OriginsInfo discussed the importance of neighbourhoods and how much information can be abstracted about people from the neighbourhoods in which they live. Webber demonstrated the importance of neighbourhood effect in various situations, including performance of schoolchildren and voting behaviour. While digital data can deliver highly specific, up-to-date details about consumers, neighbourhood data can supply contextual effects. He provided a number of examples of 'context' and concluded that digital and contextual sources should be used in combination, as both contribute towards consumer decisions.

\section{Summary}

In summary, this was a fascinating, multifaceted day with a particular focus on open data. The key messages that came through were on the great potential of open sources - data inputs are best used in combination with one another and are best selected on a 'fit-for-purpose' basis. Finally, the Census continues to provide a number of unique views of the population that are unavailable at present from any other sources, and neighbourhood contextual effects remain important - even in the current era of big data.

An earlier version of this review exists as a report on the Harnessing Open Data for Business Advantage seminar and can be accessed here: http://www.mrs.org.uk/page/8448.

Barry Leventhal

\section{IDM Data Council discussion - Consent and convenience in data collection}

Journal of Direct, Data and Digital Marketing Practice (2015) 16, 238-240. doi:10.1057/dddmp.2015.17

At the most recent session of the IDM Data Council on 4 February, there was an open discussion around the nature of the value exchange between 